\title{
The Lithium Test for Multiple Populations in Globular Clusters: Lithium in NGC 2808
}

\author{
Francesca D’Antona ${ }^{1}$ (1), Paolo Ventura ${ }^{1}\left({ }^{\circ}\right.$, Anna Fabiola Marino $^{2,3}$, Antonino P. Milone $^{3}$, \\ Marco Tailo ${ }^{3}$, Marcella Di Criscienzo ${ }^{1}$, and Enrico Vesperini ${ }^{4}$ \\ ${ }^{1}$ INAF-Osservatorio Astronomico di Roma, Via Frascati 33, I-00078 Monteporzio Catone (Roma), Italy; francesca.dantona@inaf.it, franca.dantona@gmail.com \\ ${ }^{2}$ Research School of Astronomy and Astrophysics, ANU, Mt. Stromlo Observatory, Cotter Road, Weston, ACT 2611, Australia \\ 3 Dipartimento di Fisica e Astronomia "Galileo Galilei," Univ. di Padova, Vicolo dell'Osservatorio 3, Padova, I-35122, Italy \\ ${ }^{4}$ Department of Astronomy, Indiana University, Bloomington, IN 47401, USA \\ Received 2018 December 10; revised 2018 December 30; accepted 2019 January 4; published 2019 January 23
}

\begin{abstract}
In the globular cluster (GC) NGC 2808, a quasi-standard initial lithium abundance is derived for a red giant belonging to the "extreme" population, characterized by a large helium overabundance and by abundances of proton-capture elements typical of nuclear processing in gas at very high temperatures, where the initial lithium has been fully destroyed. The observations of lithium in such extreme cluster stars are important to test different models for the formation of multiple populations in old GCs. In the asymptotic giant branch (AGB) scenario, fresh lithium is synthesized during the initial phases of hot bottom burning which, afterwards, synthesize the other p-capture elements. We model the abundance of lithium in the ejecta of super-AGB models, finding values consistent or larger than observed in the "extreme" giant; these same models describe correctly the magnesium depletion and silicon enrichment of the extreme population of NGC 2808, so the overall agreement provides further support to the AGB scenario. In the models involving massive or supermassive stars, the lithium observed requires a mixture of the lithium-free ejecta of the polluting population with more than $40 \%$ of standard-lithium pristine gas. The extended chemical anomalies of NGC 2808 stars are thus all explained within at most $60 \%$ of the possible dilution range, the initial helium mass fraction in the ejecta should be $Y \gtrsim 0.5$, to account for the $Y_{e} \sim 0.38-0.40$ of the extreme population, and further observations of p-capture elements are needed to check the model.
\end{abstract}

Key words: stars: abundances - stars: AGB and post-AGB - stars: evolution - globular clusters: general - globular clusters: individual (NGC 2808) - nuclear reactions, nucleosynthesis, abundances

\section{Introduction}

Two main environments can be the site of the nuclear proton-capture reactions necessary to explain the chemical patterns (e.g., Carretta et al. 2009) of multiple populations in globular clusters (GCs): the gas of the "second generation" (2G) stars may have been processed either

(1) in the H-burning convective cores of "first generation" (1G) high mass (e.g., Decressin et al. 2007; de Mink et al. 2009; Bastian et al. 2013) or supermassive stars (Denissenkov \& Hartwick 2014) - hereinafter the Convective Core Hydrogen Processing (CCHP) models.

(2) or in the "Hot Bottom Burning" (HBB) high temperature layers at the base of the convective envelopes of $1 \mathrm{G}$ massive Asymptotic Giant Branch (AGB) stars (Ventura et al. 2001) and "super-AGB" stars (Siess 2010; Ventura \& D'Antona 2011) - hereinafter the AGB scenario.

In this work we reconsider two important signatures of $2 \mathrm{G}$ stars, for which the CCHP models and the AGB scenario make different predictions:

(i) The observed color-magnitude diagrams of GCs allow us to put a strict upper limit $\left(Y_{\text {in }} \sim 0.41\right.$, according to Chantereau et al. 2016) on the initial helium mass fraction in the gas forming $2 \mathrm{G}$ stars, both from the modellization of the Horizontal Branches (HBs) and from the observations of the Main Sequence (MS) and Turnoff morphologies. As for the CCHP models, it is important to point out that the phase of core-Hburning generally goes throughout full $\mathrm{H}$-exhaustion leading to extreme helium abundances significantly larger than those observed. The presence of an upper limit on the helium content thus requires very specific and ad hoc assumptions concerning an early end of the H-burning when the helium core abundance goes beyond such a value (e.g., Gieles et al. 2018). On the contrary, the helium content of massive AGB envelopes is directly linked to the second-dredge up (2DU) phase, is limited to $Y=0.35-0.38$ in the standard models (e.g., Ventura 2010; Doherty et al. 2014), and reaches up to $Y \sim 0.40$ in rotating models (Georgy et al. 2013; Choi et al. 2016).

(ii) CCHP models fully destroy lithium. When this fragile element is found in the atmospheres of $2 \mathrm{G}$ stars, the gas processed by p-capture in CCHP models must be heavily diluted with unprocessed gas still preserving its standard Population II abundance. This explanation is indeed possible for a number of cases (e.g., D'Antona et al. 2012), but it must be carefully examined for extreme $2 \mathrm{G}$ stars. In the AGB scenario, at the beginning of the HBB phase, fresh lithium is produced by the Cameron \& Fowler (1971) mechanism, and remains at very high abundances in the envelope until ${ }^{3} \mathrm{He}$ is totally consumed, and eventually it is fully burned. A quantitative and close comparison with the data to either support or rule out the model, is in general complicated, since the lithium average abundance in the ejecta is scarcely constrained, due to its strong dependence on the mass-loss rate during the lithium rich phase (Ventura \& D'Antona 2005).

In Section 2 we focus our attention on a red giant (identified with the number \#46518 in the catalog by D'Orazi et al. 2015) characterized both by the maximum helium and by the presence of lithium. D'Orazi et al. (2015) find several lithium rich giants, but \#46518 belongs to the "extreme" population — the group whose helium abundance is at the highest values —and its lithium abundance is a bare factor of two smaller than in the $1 \mathrm{G}$ giants of the same sample. 

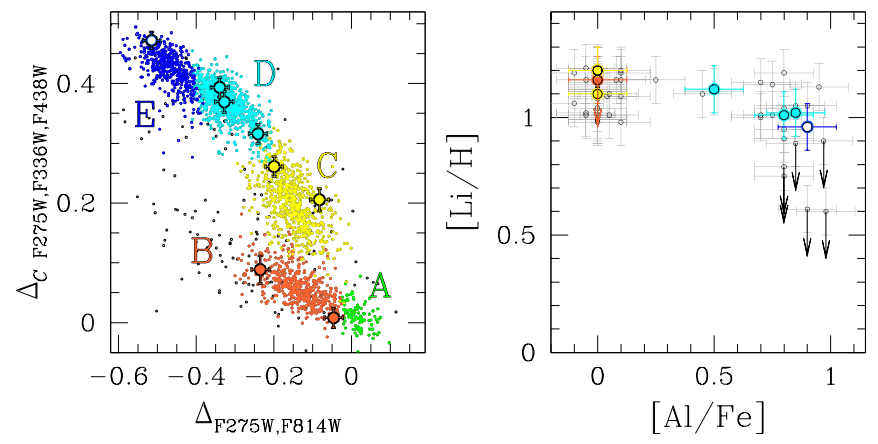

Figure 1. Left: chromosome map -location of stars in the pseudo-colors combination deviced by Milone et al. (2017) — for the giant stars in NGC 2808, subdivided into five groups from A to E by Milone et al. (2015), highlighted in different colors. Open black circles with error bars are the giants in NGC 2808 having both lithium determination and the HST photometry to locate them on the map. The giant \#46518 is shown by the black circle with white filling at the upper edge of the chromosome map, well inside the E group. Right: in the lithium vs. aluminum plane the stars of the left panel are shown in color, along with the others from the sample of D'Orazi et al. (2015).

In Section 3 we compute and analyze the detailed lithium nucleosynthesis in our super-AGB models whose yields match the other chemical anomalies in the extreme stars of NGC 2808 (Di Criscienzo et al. 2018), and confirm that the models provide the lithium and p-processing required. In Section 4 we consider the conditions under which this giant can be explained by the CCHP models, and find that tight constraints and very specific dilution patterns are required. In Section 5 we summarize the results and conclude that, with the help of future observations, the "lithium test" may result in being the ultimate tool to discriminate among formation models for GC stars.

\section{The Case of NGC 2808}

The abundance patterns of the GC NGC 2808 constitute a valuable benchmark for studying multiple populations. In NGC 2808, all the high temperature p-capture elements show large abundance variations, and the stars with different patterns of chemical abundances are subdivided into six discrete groups (Carretta 2015; Carretta et al. 2018). Also the description of this cluster in terms of "chromosome maps" (Milone et al. 2017) highlights the presence of at least five different discrete populations (named with letters from $\mathrm{A}$ to $\mathrm{E}$ in Milone et al. 2015, see Figure 1). In spite of this complexity, this cluster is indeed the best prototype of a "simple" evolution leading to the formation of multiple populations, as its stars do not show clear signs of iron spread. In the recent reanalysis of high dispersion spectroscopic data for NGC 2808, Carretta et al. (2018) point out that the detailed correlations between the different elements do not allow a simple explanation of abundances involving a single pollutor source.

\subsection{NGC 2808 in the AGB Scenario}

NGC 2808 displays a triple MS (D'Antona et al. 2005; Piotto et al. 2007) well explained by assuming three stellar populations with different helium abundances. In fact both the "blue" MS and a number of "blue hook" stars at the hot end of the HB (D'Cruz et al. 1996) indicate that NGC 2808 contains $\sim 10 \%$ of stars with a helium mass fraction $Y \simeq 0.35-0.40$ (D'Antona et al. 2005), values tantalizingly close to the maximum helium in the ejecta of AGB models. $\mathrm{X}$-shooter spectroscopy of two MS stars belonging to the standard and to the blue MS (Bragaglia et al. 2010) confirm that the blue MS star composition is typical of an extreme highly p-capture processed- population.

The triple MS found a natural explanation in the work by D'Ercole et al. (2008) exploring the formation of the $2 \mathrm{G}$ in the AGB scenario: (1) the red MS is the standard helium MS of the $1 G$; (2) the stars born from "pure" massive AGB ejecta, preserve the maximum chemical anomalies and the maximum possible helium content form the blue MS; (3) the "intermediate" MS contains stars born by gas mixture of AGB ejecta and pristine gas reaccreted from the interstellar medium. Further analysis of the chemical composition of stars in this cluster by a chemical evolution model (D'Ercole et al. 2010, 2012) were able to integrate the first proposal. The chromosome map — see Figure 1 and Milone et al. (2015) points to a more complex but similar scheme of formation of the multiple generations (D'Antona et al. 2016) in which the extreme stars (group E, blue in Figure 1) are born in a cooling flow collecting the undiluted envelopes lost by the heaviest AGB and super-AGB stars: the composition of these stars in the AGB scenario simply reflects the nucleosynthesis products of the 2DU (for the helium content) and of HBB.

\subsection{NGC 2808 in CCHP Models}

The CCHP models have some difficulty describing that $2 \mathrm{G}$ formation in NGC 2808 occurs in discrete events (Renzini et al. 2015). In addition, Carretta et al. (2018) show that the abundances of p-capture elements are not consistent with a single dilution scheme. Anyway, its extreme population is not necessarily made up of pure ejecta (see, e.g., Denissenkov \& Hartwick 2014). Uniform dilution with an $\alpha$ fraction of pristine gas may occur before second generation formation begins. In this case, it is sufficient to assume that the core-hydrogen burning stops at a helium content $Y_{\max }$ larger than the $Y_{e} \sim 0.4$ of the extreme population. Assuming $Y=0.25$ for the pristine gas, we get:

$$
Y_{\max }=\frac{Y_{e}-0.25 \alpha}{(1-\alpha)} .
$$

Notice that the initial dilution is a further hypothesis to be added in the models, as the diluting gas must in all cases contribute in the right amount to leave an $Y_{e} \sim 0.38-0.4$, similar for all the clusters hosting an extreme generation.

\subsection{Lithium in the Giants of NGC 2808}

D'Orazi et al. (2015) examined the lithium and aluminum abundance in a large group of NGC 2808 giants, less luminous than the red giant bump. The sample choice guarantees that the lithium content in the atmosphere has only suffered standard convective dilution at the first dredge up (Iben 1964), as the stars are subject to further depletion due to additional mixing mechanisms (e.g., Charbonnel \& Zahn 2007) above the red giant bump. The left panel of Figure 1 shows a few of the giants for which measurements of lithium are available on the NGC 2808 chromosome map (Marino et al. 2019; see also Carretta et al. 2018). Four stars belong either to the $1 \mathrm{G}$ population (group B, dark orange) or to group C (yellow), which formed from matter very highly diluted with pristine gas. Their $[\mathrm{Al} / \mathrm{Fe}]$ is low, confirming they belong - or have abundances similar - to $1 \mathrm{G}$, and their lithium abundance 


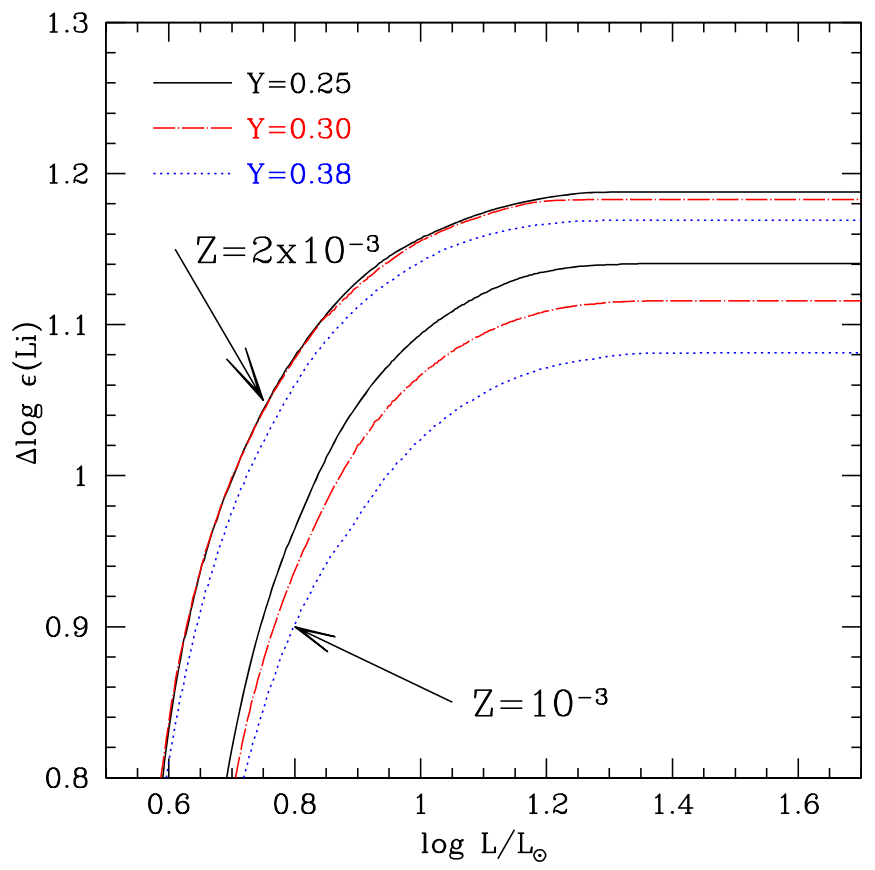

Figure 2. $\Delta \log \epsilon(\mathrm{Li})$ is the logarithmic difference between the initial abundance of lithium and the abundance along the red giant branch evolution of the masses (Table 2) evolving at 12 Gyr for different helium and metallicity. The value reaches a maximum when the first Dredge Up, at the base of the giant branch, is complete.

is $\log \epsilon(\mathrm{Li}) \sim 1-1.2^{5}$ (Figure 1, right panel). Three other stars in the D'Orazi et al. (2015) sample belong to the group D (cyan in Figure 1), a population in which the p-processed ejecta suffered intermediate dilution with pristine gas, and the giant ID\#46518, belongs to the "extreme" group (E), and has $\log \epsilon(\mathrm{Li})=0.96 \pm 0.1$. The giants with strong aluminum display either abundances slightly smaller that the $1 \mathrm{G}$ values $(\log \epsilon(\mathrm{Li}) \simeq 1)$ or much lower upper limits. We remark that the aluminum content does not allow an easy discrimination between groups D - corresponding to group I 2 in Carretta et al. (2018) - and E. In fact, although the average abundance of aluminum in the $\mathrm{E}$ group is given as $[\mathrm{Al} / \mathrm{Fe}]=1.292 \pm$ 0.029 (their Table 3), the star-to-star abundances are very scattered (see their Figure 3) and partially superimposed to the abundances of group I2. On the other hand, the chromosome map in Figure 1 shows unambiguously that the giant \#46518 belongs to the extreme population.

We estimated the convective dilution at first dredge up in coeval models of giants with different helium content, for two different metallicities bracketing the value appropriate for NGC 2808. Figure 2 and Table 1 show that the lithium depletion depends slightly on $Y$ and $Z$, at a level $<0.1$ dex. The corrections in Table 1 are applied to the giant ID\#46518: as it belongs to the high-helium $\mathrm{E}$ group, we use the $Y=0.38$ models. We obtain for the initial lithium of the star $\log \epsilon(\mathrm{Li})=2.04$ with $\sim 25 \%$ total uncertainty. By applying the correction for $Y=0.25$, the initial abundances of the B and C population stars ( standard $Y$ ) is $\log \epsilon(\mathrm{Li})=2.2 \div 2.4$, values well compatible with the abundance measured at the surface of Population II dwarfs (Asplund et al. 2006).

\footnotetext{
5 We use the usual notation $\log \epsilon(\mathrm{Li})=\log \left(N_{\mathrm{Li}} / N_{\mathrm{H}}\right)+12$, that is the number abundance with respect to hydrogen, posing the $\mathrm{H}$ number abundance at $10^{12}$.
}

Table 1

Standard Dilution of Lithium at First Dredge Up at Age 12 Gyr

\begin{tabular}{lccccc}
\hline \hline & \multicolumn{2}{c}{$Z=10^{-3}$} & & \multicolumn{2}{c}{$Z=2 \times 10^{-3}$} \\
\cline { 2 - 3 } \cline { 5 - 6 } & $M / M_{\odot}$ & $\Delta \log \epsilon(\mathrm{Li})$ & & $M / M_{\odot}$ & $\Delta \log \epsilon(\mathrm{Li})$ \\
\hline 0.25 & 0.814 & 1.14 & 0.834 & 1.19 \\
0.30 & 0.748 & 1.12 & 0.762 & 1.18 \\
0.38 & 0.644 & 1.08 & 0.658 & 1.17 \\
\hline
\end{tabular}

Note. The evolving mass and the difference in logarithmic abundance with respect to the initial content are given for three helium values and two metallicities.

\section{Super-AGB Model Results}

\subsection{Lithium Production in AGB and Super-AGB Models}

The problem of lithium production in the envelopes of luminous AGB stars was examined more than 40 years ago, based on the chain proposed by Cameron \& Fowler (1971). In fact, the terminology "hot bottom burning" (HBB) was first used for the envelope models of AGBs (Scalo \& Ulrich 1975), in which the temperature at the bottom of the convective envelope $\left(T_{\text {bce }}\right)$ reached $T_{\text {bce }} \sim 40 \mathrm{MK}$, activating the chain ${ }^{3} \mathrm{He}(\alpha, \gamma){ }^{7} \mathrm{Be}$. Ventura et al. (2001) recognized that $T_{\text {bce }}$ could reach values much larger than $40 \mathrm{MK}$, at least in the massive low metallicity AGBs. This allows full CNO cycling and the other p-capture reactions, which characterize the composition of second generation stars in GCs (see, e.g., the discussion in D'Antona et al. 2016). Lithium yields from HBB for GC-type metallicities were provided by Ventura \& D'Antona (2008).

In their first super-AGB models, Ventura \& D'Antona (2011) found a huge lithium abundance in the ejecta (for $6.5 \leqslant M / M_{\odot} \leqslant 8$, see their Table 2). This was a consequence of the Bloecker (1995) mass-loss rate, calibrated on the population synthesis of the $L \sim 2 \cdot 10^{4} L_{\odot}$ sample of lithium rich AGBs in the Magellanic Clouds (Ventura et al. 2000) and applied to super-AGBs at $L \sim 10^{5} L_{\odot}$. As the dependence on the luminosity is very strong $\left(\propto L^{3.7}\right)$, the huge mass-loss rate did not allow time for the operation of the ON cycle, and for the p-captures on magnesium, so the resulting nucleosynthesis was not compatible with that required for the extreme second generation stars.

\subsection{Lithium Production in New Super-AGB Models}

Dealing with the problem of finding new ways to calibrate the mass-loss rate in super-AGBs, Di Criscienzo et al. (2018) adopted a modulation of mass loss based on the different quantities of dust production for different parts of the evolution. This parametric approach was sufficient to achieve the required advanced nucleosynthesis shown by the extreme stars in NGC 2808. Here we extend those computations to the lithium nucleosynthesis, for all of the details, see Di Criscienzo et al. (2018).

The results are summarized in Table 2, where $\eta$ is the Reimer's parameter entering in the Bloecker (1995) mass-loss rate formulation. The temporal evolution of different elements is shown in Figure 3 for masses $6.5,7$, and $7.5 M_{\odot}$, and highlighted for the $6.5 M_{\odot}$ as an example. In stars having such massive cores $\left(\sim 1.05 M_{\odot}\right)$ the HBB temperature increases fast after the 2DU has ended, and the lithium production stage (highlighted in gold) occurs previous to the beginning of the thermal pulses (this can be seen in Figures 4 and 5). The 
Table 2

Average Abundances in the Super-AGB Ejecta for Different Masses and Mass-loss Rates

\begin{tabular}{|c|c|c|c|c|c|c|c|}
\hline$M$ & $\eta$ & $\log \epsilon(\mathrm{Li})$ & {$[\mathrm{O} / \mathrm{Fe}]$} & {$[\mathrm{Na} / \mathrm{Fe}]$} & {$[\mathrm{Mg} / \mathrm{Fe}]$} & {$[\mathrm{Al} / \mathrm{Fe}]$} & {$[\mathrm{Si} / \mathrm{Fe}]$} \\
\hline 6.5 & .02 & 2.20 & -0.38 & 0.37 & -0.09 & 1.18 & 0.44 \\
\hline 6.5 & .01 & 2.19 & -0.56 & 0.22 & -0.35 & 1.05 & 0.48 \\
\hline 6.5 & .03 & 2.42 & -0.38 & 0.49 & -0.15 & 1.19 & 0.43 \\
\hline 7.5 & .02 & 2.82 & -0.21 & 0.48 & 0.02 & 1.02 & 0.42 \\
\hline 7.5 & .01 & 2.59 & -0.26 & 0.41 & 0.10 & 1.08 & 0.44 \\
\hline 7.5 & .03 & 2.51 & -0.36 & 0.51 & 0.02 & 1.26 & 0.43 \\
\hline
\end{tabular}

Note. Initial abundances are $[\mathrm{O} / \mathrm{Fe}]=0.4,[\mathrm{Mg} / \mathrm{Fe}]=0.4,[\mathrm{Si} / \mathrm{Fe}]=0.25$, and $[\mathrm{Na} / \mathrm{Fe}]=0,[\mathrm{Al} / \mathrm{Fe}]=0$.

lithium phase occurs well before the main phase of depletion of ${ }^{16} \mathrm{O},{ }^{23} \mathrm{Na}$, and total $\mathrm{Mg}$ (region in purple). The phase of ${ }^{28} \mathrm{Si}$ production is the longest one, because it is also due to p-captures on aluminum, as shown in detail in panel (c). The sodium peak (due to the p-captures on the ${ }^{22} \mathrm{Ne}$ dredged up) is concomitant to lithium production, while the phase of sodium depletion follows on the longer timescale of the other depletions. Thus the whole lithium production stage occurs during the first phases of evolution, and the following choices of mass loss affect the processing of the other elements (magnesium, oxygen, and sodium) and not of lithium itself.

The larger masses 7 and 7.5 have similar behavior, limited to a shorter total lifetime. The larger the mass, the lower the peak of lithium production is. The reason for this is the faster cycle of production-destruction of lithium due to the larger $T_{\text {bce }}$ in the larger masses. In spite of the peak, the lithium abundance in the ejecta is larger as the mass lost during the phase of lithium production dominates over the peak abundance reached.

\subsection{Uncertainty in the Lithium Ejecta: Changing the Mass-loss Rate}

To understand the possible variations of lithium in the ejecta, we need a global study that goes well beyond the aim of this work. We exemplify the complexity of the problem by exploring different values of $\eta$. Everything else being the same, we would expect that the lithium abundance depends linearly on the mass-loss rate (that is on the $\eta$ value). In fact the situation is more complex: the $\eta=0.02$ and $\eta=0.01$ tracks for $6.5 M_{\odot}$ do not show a simple behavior (Figure 4). The track with a smaller mass-loss rate evolves to higher luminosity, and the total lithium lost is very similar. On the contrary, the average abundance increases by 0.2 dex by increasing $\eta$ by $50 \%$. In the largest super-AGB masses (the $7.5 M_{\odot}$, see Figure 5), the initially larger mass-loss rate influences $T_{\text {bce }}$ and delays the lithium production, but the models are both cooler and less luminous, so, in the end, the total mass lost during the lithium-rich phase is smaller.

Figures 4 and 5 give a fair illustration of how many physical inputs and parameters influence the lithium production, so that these results must be taken as guidelines more than at face values. Notice that not all extreme stars may preserve the lithium of the ejecta, as additional mixing mechanisms, such as advocated by Di Criscienzo et al. (2018) to explain the large spread in oxygen among the extreme stars in NGC 2808, might begin to be activated even below the bump for very helium-rich giants (see D'Antona \& Ventura 2007).

In conclusion, we should keep in mind that the results depend too much on the details of modeling and model inputs, and we should apply a generous uncertainty to the final average abundance. Still, the models show that a result very close to what we need for the extreme giant is obtained, within a few hundreths of dex.

\section{Requirements of the CCHP Models: Dilution and Lithium in 2G Stars}

Figure 6 shows specific dilution curves for lithium (a), aluminum (b), and helium content (c) in mixtures of a fraction $\alpha$ (abscissa) of standard unprocessed gas with a fraction $(1-\alpha)$ of ejecta. Thus at $\alpha=0$ we read the abundances of undiluted ejecta from the polluters and at $\alpha=1$ we have the standard abundance of the $1 \mathrm{G}$ population. For lithium, the abundances chosen for the polluter gas are $\log \epsilon(\mathrm{Li})=-1,0,1,1.5,2,2.2$, and the $1 \mathrm{G}$ has the $\log \epsilon(\mathrm{Li})=2.3$ of Population II dwarfs. If the processed gas is not from AGB ejecta, the abundance at $\alpha=0$ will be $\epsilon(\mathrm{Li})=0$, and the red dotted-dashed line represents lithium in the mixtures. The yellow region shows the range of $\alpha$ allowing us to get the abundance of lithium in the giant \#46518 in this dilution model. The request of a minimum $\log \epsilon(\mathrm{Li}) \sim 1.9$ gives a minimum $\alpha \sim 0.4$. Requiring that helium in this extreme giant is $\sim 0.4$ implies a maximum possible dilution of $\alpha \sim 0.8$. The dilution curve for helium (panel (c)) is then defined by requiring an average $Y=0.39$ for the giant, at $0.4 \lesssim \alpha \lesssim 0.8$, and $Y=0.25$ for the $1 \mathrm{G}$ giants at $\alpha=0$. The plot shows the limiting dilution lines passing through $\alpha=0.4$ and $\alpha=0.8$. The dilution curve for aluminum (panel (b)) again is limited by the $[\mathrm{Al} / \mathrm{Fe}]=0.9 \pm$ 0.15 of our giant, and by $[\mathrm{Al} / \mathrm{Fe}]=0$ of the $1 \mathrm{G}$, and the two purple lines pass through the extremes of the allowed $\alpha$ range. The range of allowed $\alpha$ values imply a very specific range for the ejecta abundances, for helium $0.5 \lesssim Y_{\max } \lesssim 1$ and for aluminum $1.15 \lesssim[\mathrm{Al} / \mathrm{Fe}]_{\max } \lesssim 1.55$. The observed abundances should then be reproduced with a single value of alpha. We also emphasize that similar specific constraints and correlations must exist for all the abundances of all other elements, such as $\mathrm{Na}$ and $\mathrm{Mg}$, which are, unfortunately, not available for the extreme star studied here. CCHP models should, however, address this issue and possibly be further tested by future observational determinations of other elements abundances for stars in the extreme second generation group.

We tentatively show the implication of dilution by plotting the $[\mathrm{Al} / \mathrm{Fe}]$ and $Y$ of the $\mathrm{D}$ group giants at possible dilution values in the figure. We use for $Y$ the values $Y=0.32$ and $Y=0.30$ consistent with group $\mathrm{D}$, from the interpretation of the middle MS. The plot shows that these few observations do not exclude the dilution model; though, there are some difficulties in allocating the $Y$ and $[\mathrm{Al} / \mathrm{Fe}]$ abundances of the 


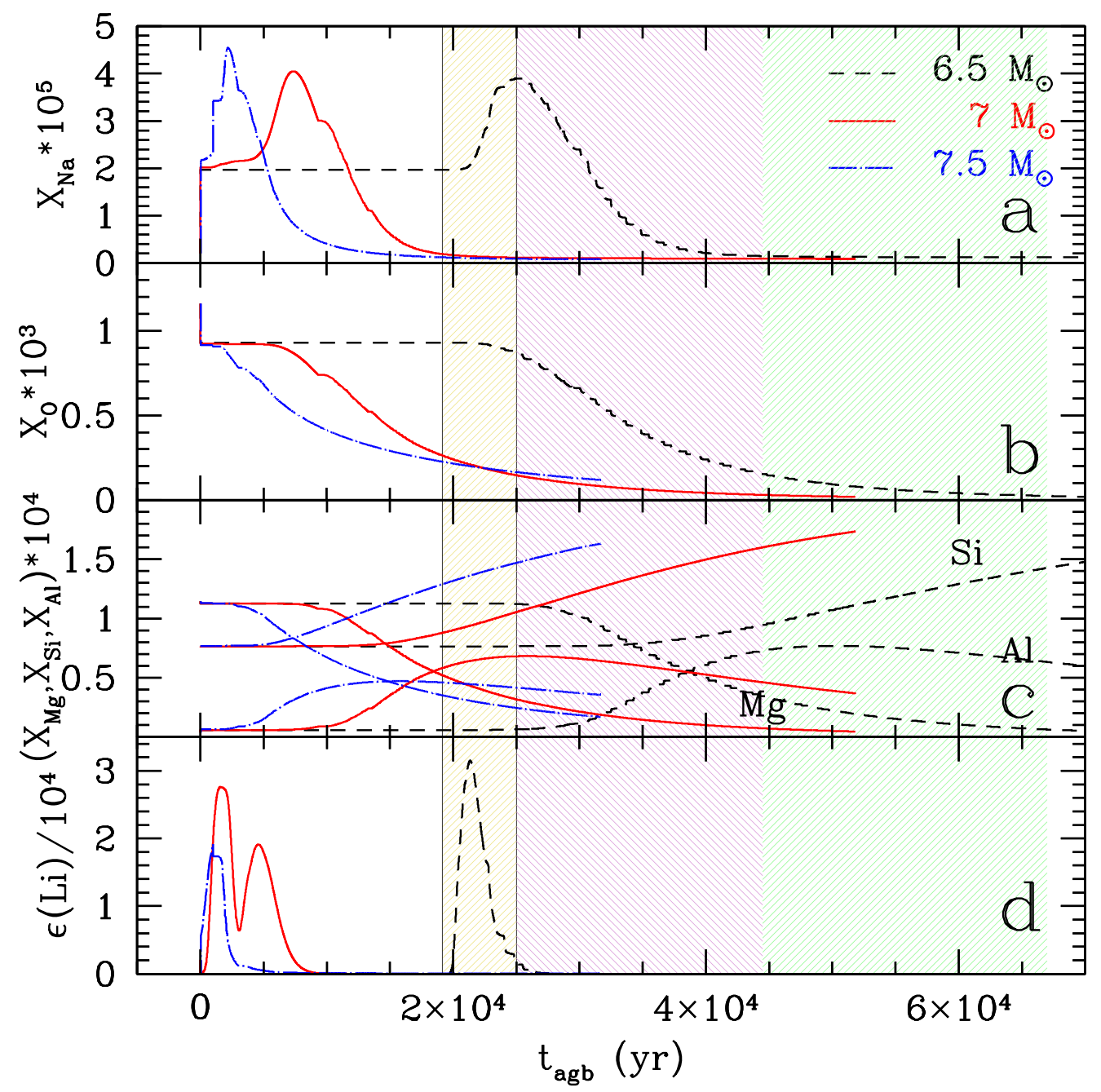

Figure 3. Evolution of the abundances of sodium (a), oxygen (b), magnesium, aluminum, and silicon (c), and lithium (d), at the surface of super-AGB stars of $6.5 M_{\odot}$ (dashed), 7 (solid line), and 7.5 $M_{\odot}$ (dashed-dotted). For the $6.5 M_{\odot}$ we highlight in different colors the time of production and destruction of lithium (gold), the main epoch of sodium, oxygen, and magnesium depletion, plus aluminum production (purple), and the longer time of final increase of silicon at the expense of magnesium and aluminum (green).

aluminum-rich $\mathrm{D}$ giants at the same dilution $\alpha$ (compare the cyan dots in panels (b) and (c)).

While Figure 6 deals with the requirements necessary to explain lithium in CCHP models, it also shows that the lithium abundance requirements in the polluting gas are not very strict in the case of the giants in group D, as the ejecta in the forming gas are well diluted with pristine gas. At $\alpha>0.4$, it is difficult to distinguish between dilution with gas deprived from lithium or dilution with gas including lithium, if the measured abundance is $\sim 2$. For this ambiguity, it is not useful to compare the lithium abundances in the giants belonging to group D, in D'Orazi et al.'s (2015) sample, with the yields resulting in the AGB models, while we considered these stars for the comparison with the CCHP models.

\section{Discussion and Conclusions}

In the framework of the chromosome maps (Milone et al. 2017) description of multiple populations in GCs, in this work we showed that a giant with quasi-standard lithium abundance (D'Orazi et al. 2015) belongs to the group E of extreme stars in NGC 2808, those having the largest possible helium abundance of $2 \mathrm{G}$ stars and the largest chemical anomalies signature of high temperature proton capture processing in the forming gas. Other stars with similar lithium belong to the less extreme group D in Milone et al. (2015). We attribute the giant \#46518 to the $\mathrm{E}$ group thanks to its unambiguous location in the pseudo-color (chromosome) maps deviced by Milone et al. (2017), because spectroscopic data are available only for lithium and aluminum, and the aluminum abundance does not allow us to easily discriminate between groups D and E, as discussed in Section 2.3.

In the AGB scenario, we identify the abundances of the E group with undiluted abundances of the ejecta of the most massive AGB and super-AGB stars (D'Ercole et al. 2008), so, if the giant \#46518 is made only by stellar ejecta, it is strictly necessary to have lithium synthesized and ejected into the intracluster medium, together with the gas very highly processed by p-captures. On the contrary, it is not necessary to explain at the same time the $\mathrm{D}$ and $\mathrm{C}$ lithium abundances, as these groups are formed from ejecta of different mass progenitors (D'Antona et al. 2016) diluted with pristine gas (see Figure 6, panel (a)). No strict correlation is required between the lithium abundance and the dilution.

To understand the giant \#46518 in the context of the AGB scenario, we computed super-AGB model evolutions. Table 2 


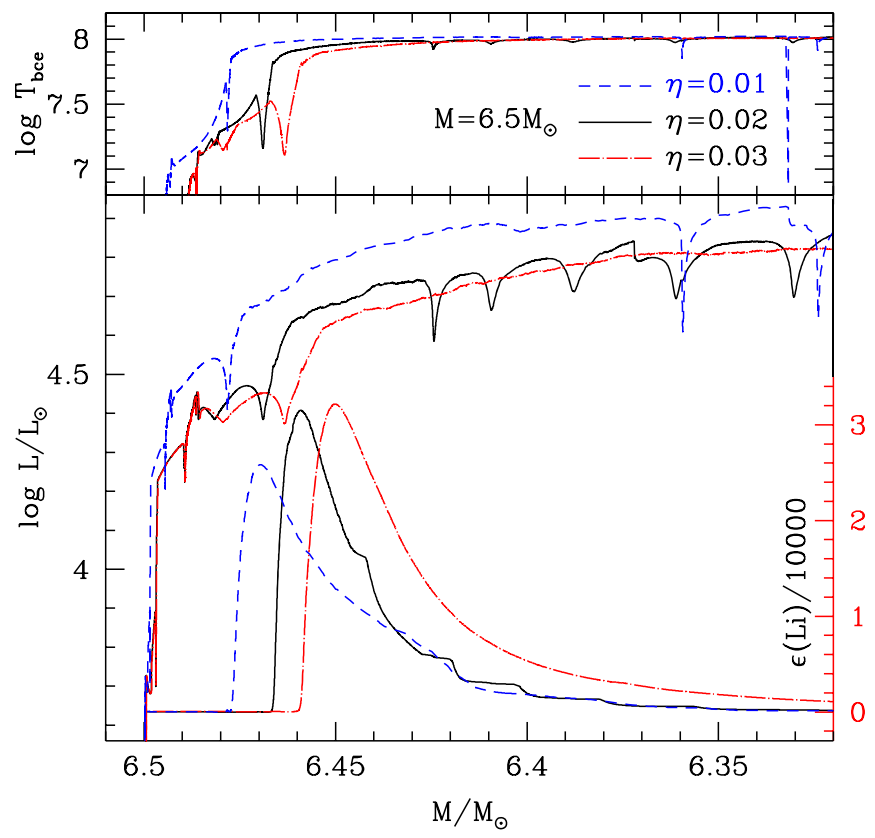

Figure 4. Evolution of the $6.5 M_{\odot}$ tracks with different mass-loss rates, $\eta=0.02$ (standard models, solid black line), $\eta=0.01$ (blue dashed), and $\eta=0.03$ (red dashed-dotted). Luminosity, lithium (bottom panel, scales on the left and on the right), and $T_{\text {bce }}$ (upper panel) are plotted vs. total stellar mass (decreasing due to mass loss), covering the phase of lithium nucleosynthesis following the $2 \mathrm{DU}$ (recognized by the minimum in $T_{\mathrm{bce}}$, followed by the onset of $\mathrm{HBB})$.

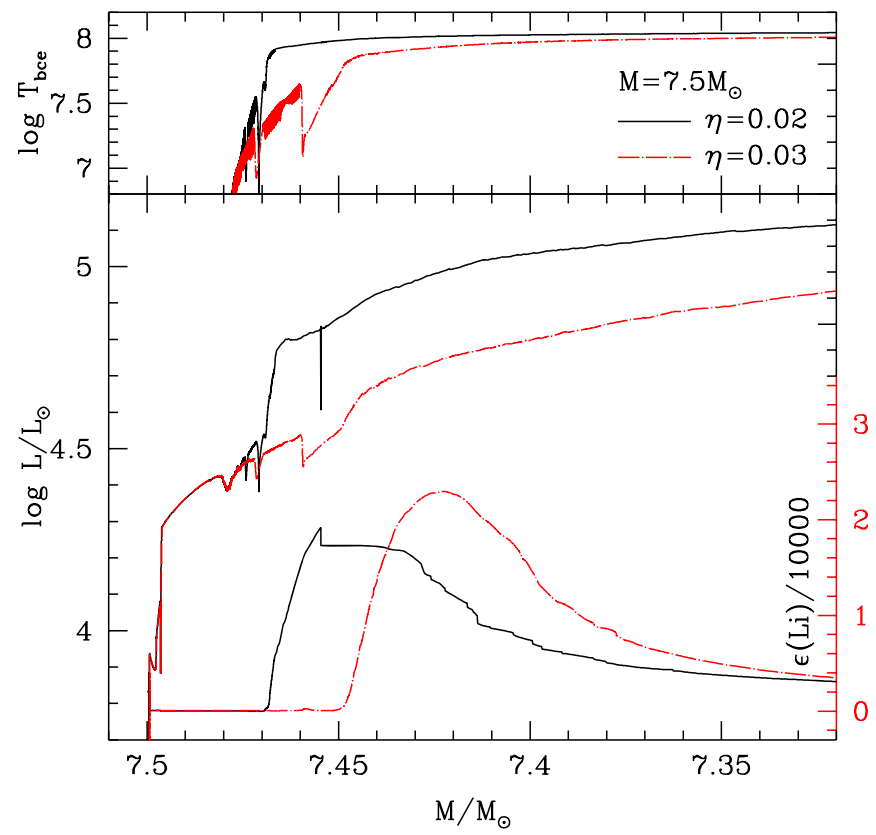

Figure 5. Same as Figure 4, for the $7.5 M_{\odot}$ track with $\eta 0.02$ (black solid line) and 0.03 (red dashed-dotted line). The maximum lithium abundance reached in the $7.5 M_{\odot}$ is smaller than in the $6.5 M_{\odot}$ (Figure 4), but the total lithium ejected is larger, because the mass-loss rate is much larger in the $7.5 M_{\odot}$.

shows that the average lithium abundances in the ejecta of the super-AGB are compatible both with the abundances of p-capture elements $(\mathrm{Mg}, \mathrm{Al}$, and $\mathrm{Si})$ in the $\mathrm{E}$ population, and with the abundance $\log \epsilon(\mathrm{Li}) \sim 1$ in the giant \#46518. In particular, it is well reproduced by the $6.5 M_{\odot}$ evolution for $\eta=0.01-0.02$, but we have shown that the detailed lithium nucleosynthesis depends on the model's detailed behavior, so

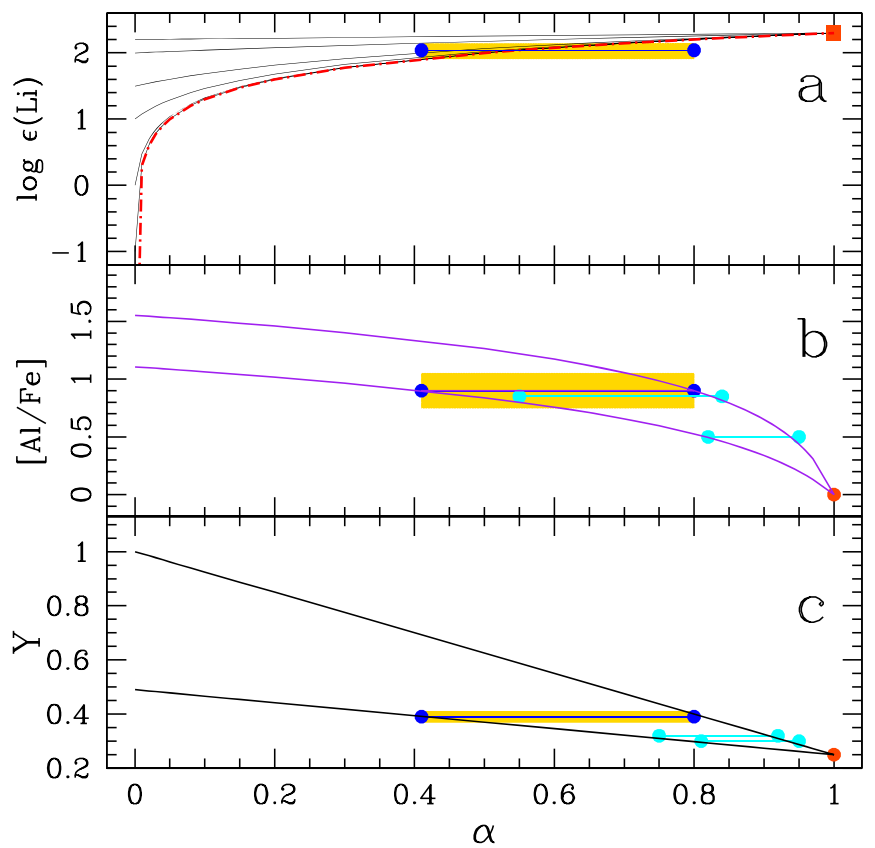

Figure 6. The abscissa is the fraction $\alpha$ of gas with standard Population II abundances, mixed with a fraction $(1-\alpha)$ of ejecta in the classic dilution scheme. In the three panels, abundances of lithium, aluminum, and helium in the mixtures are plotted. For panel (a) (lithium) the dilution curves correspond to values at $\alpha=0$ from $\log \epsilon(\mathrm{Li})=-1$ to 2.2 . The red dashed-dotted line corresponds to the fraction $(1-\alpha)$ having $\epsilon(\mathrm{Li})=0$. The point at $\alpha=1$ has $\log \epsilon(\mathrm{Li})=2.3$. The golden areas cover the $\alpha$ range allowed by the dilution model for the giant \#46518, $0.4 \lesssim \alpha \lesssim 0.8$ for lithium. Blue dots represent the extreme of the range for this giant. The cyan and red dots show the range of initial lithium in the giants of groups D and B-C, located at typical $\alpha$ for their population. Aluminum (purple, scale on the left) and (probable) helium content $Y$ (black, scale on the right) are plotted, together with the dilution curves passing through $\alpha=0.4$ and the values $[\mathrm{Al} / \mathrm{Fe}]=0$ and $Y=0.25$ at $\alpha=1$.

the agreement should be regarded as qualitative. We may expect variations of lithium, as well as of other elements among the $\mathrm{E}$ group stars, if star formation did not occur in a unique burst after accumulation in the core cooling flow of all the remnants of the super-AGB ejecta of the different masses, but in quiet star formation events as suggested by the D'Ercole et al. (2008) model. The large spread in the star-to-star abundances of p-capture elements found among group $\mathrm{E}$ stars by Carretta et al. (2018) is in fact consistent with the different abundances in Table 2 obtained from different mass progenitors, even for a single assumption on the mass-loss law. We further may expect lower lithium abundances in stars that may have suffered some extra-mixing below the bump level (Di Criscienzo et al. 2018).

Different scenarios (the CCHP models) fully destroy lithium, so they are compatible with lithium abundances only for $2 \mathrm{G}$ stars whose signatures indicate significant $(>40 \%)$ dilution with pristine matter. We remark again that Carretta et al. (2018) clearly show that a single dilution curve does not explain the whole observation set for the multiple populations of NGC 2808. Actually, they propose that the extreme population is a result of CCHP massive stellar ejecta, while the rest of $2 \mathrm{G}$ stars were born on a longer timescale in the AGB scenario. On the contrary, we focused mainly on the $\mathrm{E}$ population and concluded that the presence of a Li-rich giant and of the other typical chemical anomalies are more easily understood in the AGB scenario too. 
We have shown in previous work, at several different levels, that the AGB scenario is capable of dealing with the abundances displayed in the GC NGC 2808 (D'Ercole et al. 2008, 2010; D’Antona et al. 2016; Di Criscienzo et al. 2018), and the abundance patterns of this cluster remain a Rosetta stone that must be dealt with to falsify the models for the formation of multiple populations. Our study shows that a "Lithium test" (even at a qualitative level) can provide key insight on the origin of multiple populations and should be always considered when discussing new scenarios for the formation of multiple populations in GCs.

This work has received funding from the European Research Council (ERC) under the European Union's Horizon 2020 research innovation programme (grant Agreement ERC-StG 2016, No 716082 “GALFOR," PI: Milone), and the European Union's Horizon 2020 research and innovation programme under the Marie Sklodowska-Curie (Grant Agreement No. 797100, beneficiary: Marino). A.P.M. and M.T. acknowledge support from MIUR through the the FARE project R164RM93XW SEMPLICE.

\section{ORCID iDs}

Francesca D'Antona (i) https://orcid.org/0000-00034697-0945

Paolo Ventura (iD https://orcid.org/0000-0002-5026-6400

\section{References}

Asplund, M., Lambert, D. L., Nissen, P. E., Primas, F., \& Smith, V. V. 2006, ApJ, 644, 229

Bastian, N., Lamers, H. J. G. L. M., de Mink, S. E., et al. 2013, MNRAS, 436, 2398

Bloecker, T. 1995, A\&A, 297, 727

Bragaglia, A., Carretta, E., Gratton, R. G., et al. 2010, ApJL, 720, L41

Cameron, A. G. W., \& Fowler, W. A. 1971, ApJ, 164, 111

Carretta, E. 2015, ApJ, 810, 148
Carretta, E., Bragaglia, A., Gratton, R. G., et al. 2009, A\&A, 505, 117 Carretta, E., Bragaglia, A., Lucatello, S., et al. 2018, A\&A, 615, A17

Chantereau, W., Charbonnel, C., \& Meynet, G. 2016, A\&A, 592, A111

Charbonnel, C., \& Zahn, J.-P. 2007, A\&A, 467, L15

Choi, J., Dotter, A., Conroy, C., et al. 2016, ApJ, 823, 102

D’Antona, F., Bellazzini, M., Caloi, V., et al. 2005, ApJ, 631, 868

D’Antona, F., D'Ercole, A., Carini, R., Vesperini, E., \& Ventura, P. 2012 MNRAS, 426, 1710

D'Antona, F., \& Ventura, P. 2007, MNRAS, 379, 1431

D'Antona, F., Vesperini, E., D'Ercole, A., et al. 2016, MNRAS, 458, 2122

D’Cruz, N. L., Dorman, B., Rood, R. T., \& O'Connell, R. W. 1996, ApJ, 466, 359

Decressin, T., Meynet, G., Charbonnel, C., Prantzos, N., \& Ekström, S. 2007, A\&A, 464, 1029

de Mink, S. E., Pols, O. R., Langer, N., \& Izzard, R. G. 2009, A\&A, 507, L1

Denissenkov, P. A., \& Hartwick, F. D. A. 2014, MNRAS, 437, L21

D'Ercole, A., D’Antona, F., Carini, R., Vesperini, E., \& Ventura, P. 2012, MNRAS, 423, 1521

D’Ercole, A., D’Antona, F., Ventura, P., Vesperini, E., \& McMillan, S. L. W. 2010, MNRAS, 407, 854

D’Ercole, A., Vesperini, E., D’Antona, F., McMillan, S. L. W., \& Recchi, S. 2008, MNRAS, 391, 825

Di Criscienzo, M., Ventura, P., D’Antona, F., Dell'Agli, F., \& Tailo, M. 2018, MNRAS, 479, 5325

Doherty, C. L., Gil-Pons, P., Lau, H. H. B., et al. 2014, MNRAS, 441, 582

D'Orazi, V., Gratton, R. G., Angelou, G. C., et al. 2015, MNRAS, 449, 4038

Georgy, C., Ekström, S., Granada, A., et al. 2013, A\&A, 553, A24

Gieles, M., Charbonnel, C., Krause, M. G. H., et al. 2018, MNRAS, 478, 2461

Iben, I., Jr. 1964, ApJ, 140, 1631

Marino, A. F., Milone, A. P., Renzini, A., et al. 2019, ApJ, submitted

Milone, A. P., Marino, A. F., Piotto, G., et al. 2015, ApJ, 808, 51

Milone, A. P., Piotto, G., Renzini, A., et al. 2017, MNRAS, 464, 3636

Piotto, G., Bedin, L. R., Anderson, J., et al. 2007, ApJL, 661, L53

Renzini, A., D'Antona, F., Cassisi, S., et al. 2015, MNRAS, 454, 4197

Scalo, J. M., \& Ulrich, R. K. 1975, ApJ, 200, 682

Siess, L. 2010, A\&A, 512, A10

Ventura, P. 2010, in IAU Symp. 268, Light Elements in the Universe, ed. C. Charbonnel et al. (Cambridge: Cambridge Univ. Press), 147

Ventura, P., \& D'Antona, F. 2005, A\&A, 431, 279

Ventura, P., \& D'Antona, F. 2008, A\&A, 479, 805

Ventura, P., \& D'Antona, F. 2011, MNRAS, 410, 2760

Ventura, P., D’Antona, F., \& Mazzitelli, I. 2000, A\&A, 363, 605

Ventura, P., D’Antona, F., Mazzitelli, I., \& Gratton, R. 2001, ApJL, 550, L65 